\title{
Erratum: Quorum-sensing active particles with discontinuous motility [Phys. Rev. E 101, 012601 (2020)]
}

\author{
Andreas Fischer, Friederike Schmid, and Thomas Speck (]
}

(Received 2 November 2020; published 17 November 2020)

DOI: 10.1103/PhysRevE.102.059903

In deriving the density and polarization profiles, and motivated by the numerical simulations, we had posited that the polarization within the passive cluster is zero, $p=0$, and jumps to a nonzero value outside [cf. inset of Fig. 3]. However, this is only the case for a special situation (to which we come back below). In analogy with electrostatics, a continuity condition had been derived for the quantity (equations are numbered as in the original paper),

$$
M_{0}=-\frac{1}{2} v \rho+D_{0}\left(1+v^{2}\right) \partial_{x} p
$$

now restricted to a planar interface with density $\rho(x)$, polarization $p(x)$, and speed $v$ jumping at the motility interface at $x=0$. We employ the abbreviation $v=v_{0} / v_{*}$. As long as $M_{0}$ is finite the polarization clearly has to be continuous at variance with our assumption.

We now derive the corrected expressions. For the planar interface, the inner solution reads $p^{<}(x)=a e^{x / \ell}$ whereas the outer solution still is $p^{>}(x)=a e^{-x / \xi_{0}}$. The jump of the first derivative is given by the continuity condition $M_{0}^{<}=M_{0}^{>}$reading

$$
\left.D_{0} \partial_{x} p^{<}\right|_{0}=-\frac{1}{2} v_{0} \rho_{c}+\left.D_{0}\left(1+v^{2}\right) \partial_{x} p^{>}\right|_{0} .
$$

We, thus, have to replace Eq. (21) by

$$
-D_{0} \frac{a}{\ell}=\frac{1}{2} v_{0} \rho_{c}+D_{0}\left(1+v^{2}\right) \frac{a}{\xi_{0}}
$$

leading to the polarization at the interface,

$$
a=-\frac{1}{2} \frac{v_{0} \xi_{0}}{D_{0}}\left(1+v^{2}+\xi_{0} / \ell\right)^{-1} \rho_{c}<0,
$$

that differs by the term $\xi_{0} / \ell$ inside the brackets. This affects the following Eq. (22), which is to be replaced by

$$
\frac{\rho_{g}}{\rho_{c}}=1-8 f
$$

defining the function,

$$
f(v)=\frac{v^{2}\left(1+v^{2}\right)}{\left(1+8 v^{2}\right)\left(1+v^{2}+\xi_{0} / \ell\right)} .
$$

The following equations are correct although the threshold is affected after inserting $a$ and reads explicitly

$$
\frac{\bar{c}}{c_{0}}=\left[1-4 f\left(1-\frac{2}{\pi} \frac{\cos ^{-1}\left(\lambda / \xi_{0}\right)}{\sqrt{1-\left(\lambda / \xi_{0}\right)^{2}}}\right)\right] \frac{\rho_{c}}{\rho_{0}} .
$$

Figure 1 shows the corrected active gas densities $\rho_{c}$ and dense passive densities $\rho_{c}$ as a function of $\nu$, cf. Fig. 3(a) in the original paper. Although there is a quantitative change, the conclusions of the original paper are not affected by this.

The same argument holds for the circular clusters, also here the polarization inside the cluster is, in general, nonzero and given by $p^{<}(r)=a_{1}^{<} I_{1}(r / \ell)$ with the modified Bessel function $I_{n}(x)$ of order $n$. The continuity condition now reads

$$
\left.D_{0} \partial_{r} p^{<}\right|_{r_{c}}=-\frac{1}{2} v_{0} \rho_{c}+\left.D_{0}\left(1+v^{2}\right) \partial_{r} p^{>}\right|_{r_{c}}
$$

and, thus,

$$
-D_{0}\left(\frac{a_{1}^{<}}{\ell} I_{0}\left(r_{c} / \ell\right)-\frac{p_{c}}{r_{c}}\right)=\frac{1}{2} v_{0} \rho_{c}+D_{0}\left(1+v^{2}\right)\left[\frac{a_{2}^{>}}{\xi_{0}} K_{0}\left(r_{c} / \xi_{0}\right)+\frac{p_{c}}{r_{c}}\right],
$$




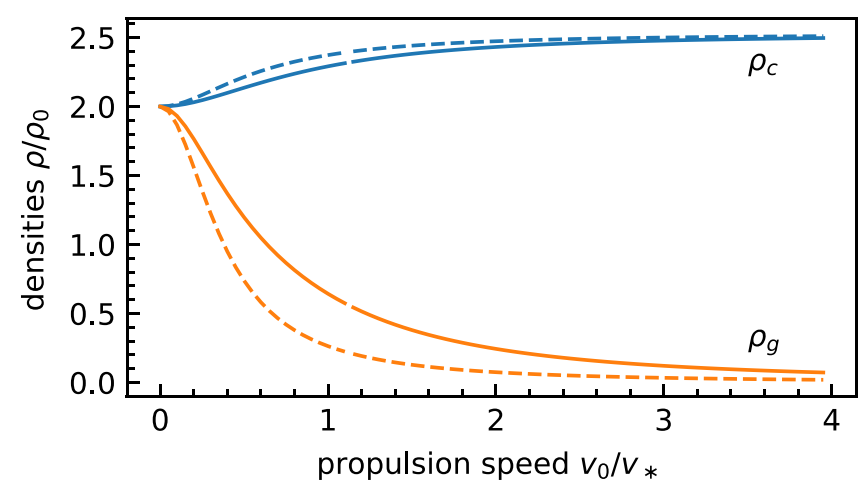

FIG. 1. Coexisting densities of active gas $\left(v=v_{0}\right.$, bottom) and passive cluster ( $v=0$, top). The dashed lines are the results for a jump of the polarization whereas the solid lines are the corrected values for a continuous polarization across the motility interface.

with the polarization,

$$
p_{c}=a_{1}^{<} I_{1}\left(r_{c} / \ell\right)=a_{2}^{>} K_{1}\left(r_{c} / \xi_{0}\right),
$$

at the interface. Using Eq. (29) to eliminate

$$
\frac{a_{2}^{>}}{\xi_{0}} K_{0}\left(r_{c} / \xi_{0}\right)=-\frac{D_{0}}{v_{0} \ell^{2}} \frac{1+8 v^{2}}{1+v^{2}}\left(\rho_{c}-\rho_{g}\right)
$$

we then have to replace Eq. (32) by

$$
\rho_{c}=\left(1+8 v^{2}\right) \rho_{g}+\frac{v_{0}}{D_{r}}[\underbrace{\frac{r_{c}}{\ell} \frac{I_{0}\left(r_{c} / \ell\right)}{I_{1}\left(r_{c} / \ell\right)}}_{g\left(r_{c} / \ell\right)}+v^{2}] \frac{p_{c}}{r_{c}},
$$

wherein the function $g$ was unity before.

The simulations have been performed with interacting repulsive particles modeling volume exclusion. The diffusion coefficient of the passive particles then becomes much smaller than the bare diffusion coefficient $D^{<} \ll D_{0}$. This means that the decay length $\ell$ is also small. The derivative of the inner solution for the polarization,

$$
\frac{1}{\ell} e^{x / \ell} \stackrel{\ell \rightarrow 0}{\longrightarrow} \delta(x)
$$

then approaches a Dirac- $\delta$ distribution. In that limit $M_{0}$ is no longer finite at the motility interface and the polarization does jump (an electrostatic analog is a dipole layer with an infinite field causing a jump of the electrostatic potential). Although not correct, in general, we, thus, find that a jump of the polarization is still a good approximation for the numerical simulations (and experiments [1]) we have performed. The discrepancies between mean-field theory and simulations are rather due to interfacial fluctuations as discussed in detail in the paper.

We thank S. Auschra and N. A. Söker for drawing our attention to this issue, see Refs. [2,3] for their approach to motility interfaces.

[1] T. Bäuerle, A. Fischer, T. Speck, and C. Bechinger, Self-organization of active particles by quorum sensing rules, Nat. Commun. 9, 3232 (2018).

[2] N. A. Söker, S. Auschra, V. Holubec, K. Kroy, and F. Cichos, Active-particle polarization without alignment forces, arXiv:2010.15106.

[3] S. Auschra, V. Holubec, N. A. Söker, F. Cichos, and K. Kroy, Polarization-density patterns of active particles in motility gradients, arXiv:2010.16234. 